\title{
Linx
}

Revue des linguistes de l'université Paris X Nanterre

$7 \mid 1995$

Saussure aujourd'hui

\section{Saussure ou la pensée comme représentation}

\section{Gabriel Bergounioux}

\section{OpenEdition}

\section{Journals}

Édition électronique

URL : http://journals.openedition.org/linx/1145

DOI : 10.4000/linx. 1145

ISSN : 2118-9692

\section{Éditeur}

Presses universitaires de Paris Nanterre

\section{Édition imprimée}

Date de publication : 1 septembre 1995

Pagination : 173-186

ISSN : 0246-8743

\section{Référence électronique}

Gabriel Bergounioux, «Saussure ou la pensée comme représentation », Linx [En ligne], 7 | 1995, mis en ligne le 13 juillet 2012, consulté le 19 avril 2019. URL : http://journals.openedition.org/linx/1145; DOI : 10.4000/linx.1145

Ce document a été généré automatiquement le 19 avril 2019.

Département de Sciences du langage, Université Paris Ouest 


\title{
Saussure ou la pensée comme représentation
}

\author{
Gabriel Bergounioux
}

1 Alors que la question du sens fait retour dans une reformulation cognitiviste, c'est-à-dire comme une énième version du renoncement à l'autonomie du langage au profit de procédures psychologisantes, il nous paraît que l'enseignement de Saussure spécialement son (problématique) rappport à la sémantique et à la psychologie-, argumente une critique à l'oeuvre dans les textes. Encore ceux-ci requièrent-ils une triple perspective de lecture :

- historique: à quelles références, quelles interprétations et quels enjeux répond la conceptualisation saussurienne?

- exégétique : le Cours de Linguistique Générale ${ }^{1}$, eu égard au caractère très particulier de son établissement et de ses versions, est-il univoquement interprétable?

- éristique : quelle charge critique emporte jusqu'à aujourd'hui la tentative de Saussure de penser la langue (et la linguistique) en rupture avec la pensée de ses contemporains ?

\section{Saussure et la psychologie de son temps}

2 A partir de 1880, la configuration scientifique du marché de la linguistique polarise toute volonté de dépassement des néo-grammairiens soit vers quelque découverte épigraphique qui infirmerait la rigidité schématique de l'évolution postulée dans les langues indoeuropéennes, et c'est le déchiffrement du hittite, du tokharien, soit vers un recours à quelque nouveau paradigme, particulièrement la psychologie.

3 La voie, ouverte par Wundt (1893) ${ }^{2}$, est reprise et amplifiée, en Angleterre grâce aux travaux de Galton, en France par les recherches sémantiques de Darmesteter et Bréal, en Allemagne par l'école expérimentale. On retrouverait les tentatives de van Ginneken (1907), de Sechehaye (1908) et pourquoi pas de Saussure lui-même :

«psychologie: très difficile de marquer la séparation de la langue avec elle. Tout est psychologique dans la linguistique, y compris ce qui est mécanique et matériel (changements de sons, etc.). » 
si ce n'est cette réserve ${ }^{4}$ :

«On a prétendu que la linguistique rentre directement dans la psychologie et attend d'elle ses lumières. Or la psychologie possède-t-elle une sémiologie? La question est inutile, vu que si elle en possédait une, les phénomènes de la langue, que la psychologie ignore, seraient tellement prépondérants à eux seuls, comme base du fait sémiologique, que tout ce qui aurait pu être dit hors d'eux par la psychologie ne représente forcément rien ou à peu près rien. »

Encore reste-t-il à déterminer quelle idée Saussure se faisait de la psychologie après que Michel Arrivé a démontré combien la genèse du Cours demeurait indifférente au freudisme ${ }^{5}$. En schématisant (mais la vision de Saussure a quelque chance d'avoir été aussi schématique), le choix se limitait à quatre écoles :

- l'école clinique française, établie par Broca et imposée par Charcot, dont les prétentions se fondent sur le "parallélisme»: toute manifestation physique correspond à une activité psychique distincte, et réciproquement.

- l'école allemande, dite "expérimentale », représentée par Wundt et ses élèves, notamment Thumb et Marbe ;

- l'école anglaise, dite "associationniste», illustrée par les deux Mill, Spencer (en France, Taine) et, à son corps défendant, W. James ;

- l'école spiritualiste française dont la filiation, de Maine de Biran au premier Bergson passe par La parole intérieure de Victor Egger ${ }^{6}$;

5 En dépit de références éparses à un «langage intérieur»-formulation d'ailleurs contradictoire avec son ordinaire terminologie-, Saussure voit deux raisons pour refuser l'approche introspectionniste de Victor Egger. D'abord, elle n'offre pas accès aux principes du fonctionnement de la langue mais seulement à son exercice (la parole) ; ensuite, elle privilégie l'observation d'un individu au détriment de l'analyse d'un mécanisme $^{7}$ :

« En second lieu, on est porté, quand on veut approfondir le signe, à établir les opérations mentales et physiques saisissables chez l'individu à propos du signe. Pourquoi choisit-on l'individu? Parce qu'ainsi nous sentons les choses à notre portée. Or cela n'est que l'exécution du signe. On passe donc à côté. »

Cette remarque récuse, sous une même critique et pour la même raison de méthode, l'idéalisme d'Egger, l'école expérimentale allemande, le behaviourisme et l'école clinique de Broca et Charcot. De cette dernière, Saussure se démarque plus nettement quand il se défend d'une comparaison entre versant matériel et « spirituel » de la langue ; alors qu'il cherche à quoi comparer l'appariement du signifiant et du signifié, d'un mot qui détone, par sa familiarité, avec la tenue ordinaire du style, il déclare ${ }^{8}$ :

«Le corps et l'âme, faisant la personne, font une comparaison -qui cloche, du reste.»

«La comparaison la plus ressassée, c'est la comparaison du corps et de l'âme, qui font la personne. Mais cette comparaison cloche par bien des points. »

7 C'est Charcot qui est visé, pour avoir, dans les années 80 , en réponse à La parole intérieure de Victor Egger, proposé une représentation des fonctions mentales à partir d'une clinique de l'aphasie9. Le schéma qu'il impose, et qu'il dit justifier par les localisations cérébrales, subsume à un centre d'idéation quatre types de processus (articulatoire, graphique, visuel et auditif) articulés aux organes sensori-moteurs. Cette doxa circule sous forme d'un croquis, tracé par le maître, représentant, radiant autour d'un cercle (le centre d'idéation) quatre rayons terminés par des lèvres, une écriture en cursives, une 
écriture d'imprimerie et une oreille, les quatre formes sous lesquelles pourrait être actualisée, pour un sujet, l'idée de « cloche » (prononcée, écrite, lue et entendue).

De ces quatre formes, Saussure -il ne variera jamais sur ce point-, n'en reconnaît qu'une : celle que, du côté de l'auditif, il nomme l'image acoustique. Il n'existe aucun usage, dans la représentation qu'il donne de la langue, pour un lieu de pure pensée auquel on accèderait par le détour des mots. Les mots, dans leur forme sonore, sont la pensée même. C'est cette thèse qui le sépare de la psychologie expérimentale allemande, de la philosophie spiritualiste française et de la pseudo-clinique de Charcot.

Il est dès lors commode de rattacher Saussure à la quatrième école, l'associationnisme, comme l'usage constant de ce terme dans le Cours y invite ; pourtant, c'est l'inverse que nous soutiendrons : que Saussure promeut de l'association une théorie qui est la négation de l'associationnisme au sens qu'a reçu ce concept dans la philosophie du XIXe siècle.

\section{De l'association a l'union}

L'usage hétérodoxe de Saussure quand il recourt au terme d'association a été perçu par Benveniste qui esquive le mot dans un hommage ${ }^{10}$ centré sur l'invention d'une « unité à double face ». Le problème est rendu plus complexe du fait que association renvoie à deux relations assez différentes :

- l'association, au singulier, désigne le rapprochement du signifiant et du signifié, une association externe puisqu'elle concerne des éléments hétérogènes ;

- les associations, au pluriel, ou mieux : les rapports associatifs -la nuance est d'importances'opposent aux rapports syntagmatiques et il s'agit d'une association interne, articulant des éléments homogènes.

11 Saussure fait le rapprochement dans le premier de ses cours ${ }^{11}$ :

«Le classement intérieur. [Si] la masse de formes qui composent la langue pour chaque individu ne [restait qu'] un chaos dans chaque tête [la parole et la langue seraient inconcevables]. La nécessité d'un classement, d'un ordre quelconque est une nécessité a priori, même sans mettre en avant la psychologie. Comme premier élément de cet ordre nous devons poser : l'association primordiale entre forme et idée et groupes d'idées; puis une autre association sans laquelle la première ne pourrait [pas] exister : l'association de forme à forme, des formes entre elles. »

12 Néanmoins, l'association du signifiant et du signifié ne constitue pas une association telle que la définirait l'associationnisme: les deux éléments qui constituent le signe -et qu'après bien des hésitations Saussure baptise « signifiant » et « signifié » - ne préexistent pas au terme qui les somme.

13 Benveniste, tordant le bâton dans l'autre sens, suggère que l'opération d'associer précéderait les termes associés. Nous y verrions plutôt, par analogie avec ce que la psychanalyse a pensé comme "après-coup", une totalité composée d'éléments qui n'existent que pour autant qu'ils constituent cette totalité ; une fois le signe établi, les éléments constitutifs ne sont plus "perceptibles» (n'existent plus) hors de la totalité qu'ils font exister.

On comprend mieux, devant cette définition circulaire, l'embarras de Saussure pour caractériser ce qui pré-existerait aux signes et combien il répugne à préciser ce que seraient les idées et les sons avant d'accéder au statut de signifiants et de signifiés. Pour surmonter la distance qui les sépare, il n'a d'autre recours qu'une référence à l'esprit : 
«(...) le signe linguistique repose sur une association faite par l'esprit entre deux choses très différentes $(. ..) \|^{12}$ débarrassée du signifiant (et de la syntaxe), Saussure soutient qu'il n'est qu'une écriture qui soutienne la spécificité du langage articulé: l'alphabet dans sa représentation linéaire. Qu'une figuration "idéogrammatique» (ou "pasigraphique ») puisse représenter quoi que ce soit de pertinent dans l'ordre du langage, et de la pensée, est contradictoire avec les principes du Cours. Saussure pointe là le défaut constitutif du projet sémantique qui perd toute validité en écartant, a priori, la qualité signifiante et syntagmatique des signes, renouvelant l'erreur par où la psychologie s'est condamnée à manquer son objet. Il le dit, sans ménagement, quand un de ses élèves lui semble se détourner de son enseignement ${ }^{16}$ :

"J'ai indiqué mes critiques. En somme, M. Sechehaye, après avoir reproché à Wundt, avec raison, d'avoir méconnu le problème grammatical, arrive lui-même à ne pas s'en faire une idée suffisante. Car la seule idée suffisante serait de poser le fait grammatical en lui-même, et dans ce qui le distingue de tout autre acte psychologique, ou en outre logique. Plus l'auteur prend de peine à abattre ce qui lui semble une barrière illégitime entre la forme pensée et la pensée, plus il nous semble s'éloigner de son propre but, qui serait de fixer le champ de l'expression, et d'en concevoir les lois, non dans ce qu'elles ont de commun avec notre psychisme en général, mais dans ce qu'elles ont au contraire de spécifique et d'absolument unique, dans le phénomène de la langue.»

Aujourd'hui, la sémantique cognitive, dans sa visée et ses applications, ne nous semble pas en reste dans ce fourvoiement (elle aurait même plutôt de l'avance). 


\section{Des rapports associatifs : le processus e(s)t le resultat}

21 Après l'association du signifiant et du signifié, voyons les associations -ou plutôt les rapports associatifs. Cette notion vient, dans les leçons de Saussure, sitôt qu'il énonce les partitions élémentaires qu'une linguistique doit construire pour être adéquate à son objet. De l'opération elle-même, « associer », Saussure constate qu'elle est double ${ }^{17}$ :

« Associer deux formes, ce n'est pas seulement sentir qu'elles offrent quelque chose de commun, c'est aussi distinguer la nature des rapports qui régissent les associations. "

Deux opérations sont confondues dans l'acte d'associer: d'une part, deux éléments qui présentent un élément commun spontanément perçu par le locuteur (notamment dans la chaîne phonétique) sont rapprochés; d'autre part, la nature du rapport associatif actualisé est catégorisée. Partant des connaissances subjectives implicites, le linguiste explicite la catégorisation dans des formes de connaissance universelle (scientifique) ${ }^{18}$.

Associer ne prend sens que dans l'opposition structurale: associer vs coordonner. Construire simultanément ces deux opérations, énoncées à la fin des partitions initiales du Cours (p. 29), caractérisées ultérieurement comme «deux formes de notre activité mentale ( Cours p.170), emporte rétroactivement une incidence: l'activité que l'on désigne comme «langue » n'est pas primaire (ou élémentaire) puisqu'elle résulte de la combinaison de deux facultés (associer et coordonner), inséparables dans la langue.

Ces deux types de rapports s'opposent comme suit ${ }^{19}$ :

\begin{tabular}{|l|l||l|}
\hline & « rapports associatifs » & « rapports syntagmatiques » \\
\hline & « virtuels & « effectifs » \\
\hline & « in absentia & « in praesentia » \\
\hline & « intuitif» & « discursif. \\
\hline
\end{tabular}

Ce que Saussure nomme rapports associatifs diffère de ce que conceptualisait comme association la tradition philosophique et psychologique anglaise en ce que :

- l'opération n'est pas distinguée du résultat (il n'y a pas d'un côté l'opération et de l'autre la somme), ce qu'indique la prédilection pour le syntagme « rapports associatifs $»^{20}$;

- ces rapports ne sont pas un cas particulier de l'association psychologique mais l'une des deux dimensions fondatrices du langage ;

- les éléments n'en sont pas sommables.

Concernant le dernier point, Saussure a tergiversé avant de risquer l'énoncé des deux propriétés caractéristiques des rapports associatifs (c'est-à-dire le réseau dans lequel une unité acoustique, segmentée par recours au sens, reçoit une valeur) :

- les rapports associatifs ne sont pas en nombre défini ;

- ils ne se présentent pas en ordre déterminé.

"Tandis qu'un syntagme appelle tout de suite l'idée d'un ordre de succession et d'un nombre déterminé d'éléments, les termes d'une famille associative ne se présentent ni en nombre défini, ni dans un ordre déterminé. ${ }^{21}$ 
une rupture radicale avec les psychologies associationniste et expérimentale. Selon Saussure, leurs résultats n'ont de validité qu'en fonction des expériences proposées, qui visent à connaître la parole, sans qu'aucune conséquence générale puisse en être tirée concernant le fonctionnement de la langue.

Ce considérant nous paraît être la ligne de rupture irréversible de la linguistique saussurienne contre toutes les formulations normatives d'une orthopédie verbale et mentale ${ }^{22}$. Saussure invalide le principe même de recherches associatives fondées sur une hypostase du schéma «Stimulus-Réponse », il refuse l'objectivation par chronomètres et statistiques, et sa désagréable (même, inquiétante) visée de conformisme ${ }^{23}$. Il n'y a pas de modèle, d'idéal, d'archétype universalisable dans le processus d'association, seulement un rapprochement toujours variable d'éléments :

"Si on associe désir-eux, chaleur-eux, peur-eux, etc., on ne saurait dire d'avance quel sera le nombre des mots suggérés par la mémoire, ni dans quel ordre ils apparaîtront. $»^{24}$

selon des points de vue qui ne varient pas moins (Cours, p. 173) :

«Les groupes formés par association mentale ne se bornent pas à rapprocher les termes qui présentent quelque chose de commun ; l'esprit saisit aussi la nature des rapports qui les relient dans chaque cas et crée par là autant de séries associatives qu'il y a de rapports divers. "

Et Benveniste n'a pas manqué, dans son hommage, de relever ce propos de Saussure :

"Voici notre profession de foi en matière linguistique : en d'autres domaines, on peut parler de choses à tel ou tel point de vue, certain qu'on est de retrouver un terrain ferme dans l'objet même. En linguistique, nous nions en principe qu'il y ait des objets donnés, qu'il y ait des choses qui continuent d'exister quand on passe d'un ordre d'idées à un autre. $»^{25}$

Donc, il n'y a pas d'ordre naturel, ou seulement meilleur, parce que les éléments sont à chaque instant construits selon un point de vue qui privilégie une identification (de forme, de fonction ou de sens) qui ne garantit pas même l'identité à soi, fondement de toute psychologie ${ }^{26}$ :

«Lorsque, dans une conférence, on entend répéter à plusieurs reprises le mot Messieurs!, on a le sentiment qu'il s'agit chaque fois de la même expression, et pourtant les variations de débit et l'intonation la présentent, dans les divers passages, avec des différences phoniques très appréciables (...); en outre, ce sentiment de l'identité persiste, bien qu'au point de vue sémantique non plus il n'y ait pas identité absolue d'un Messieurs ! à l'autre, de même qu'un mot peut exprimer des idées assez différentes sans que son identité soit sérieusement compromise (cf «adopter une mode » et « adopter un enfant », « la fleur du pommier » et « la fleur de la noblesse », etc.).»

31 Cette réfutation du behaviourisme, qui pourrait concerner par principe toute sémantique, se fonde sur le fait que les processus linguistiques ne sont pas assimilables à un choix entre des possibles pré-existants, parmi lesquels l'homoncule (dixit Lacan) opterait. Il n'y a pas choix, seulement construction de rapports associatifs.

Mais cette conception des rapports associatifs revient à récuser aussi le fantasme mécaniste, hypostasiant l'auto-organisation d'une langue qui inclurait, dans sa disposition structurale, tous les principes d'une organisation suffisante qu'il ne resterait plus qu'à mettre au jour (i.e. pré-construite). On reconnaîtra là, notamment, ce qui a reçu une consécration figée, arthritique dans la notion de "paradigme " dont on affecte d'oublier qu'elle ne figure qu'une seule fois dans le Cours, comme synonyme de déclinaison. A vouloir le généraliser, le concept de paradigme serait au mieux un rapport 
associatif parmi d'autres; il est vrai qu'on a confondu sous ce nom, et en excipant de la " commutation", des faits aussi disparates que les variations morphématiques (flexion, marques de nombre, etc.), sémantiques et même phonétiques (en poétique notamment).

Contre cette conception du paradigme, rappelons ce propos ${ }^{27}$ :

«(...) ce qui va autour de lui [le mot] associativement, cela n'est dans aucun

contexte, vient de la conscience (...) ",

dans lequel la conscience définit un lieu ( le lieu des "associations») et non un schéma opérateur. Ce qui laisse en suspens la question centrale de la psychologie : le statut du sujet.

\section{Ce que savent les sujets}

S'il n'est d'entités dans la langue que construites (ou, ce qui revient au même, si la langue n'est pas composée d'entités pré-existantes, en nombre fini et dans un ordre donné), comment l'objectiver? Saussure propose d'abord de recourir au réel (concret) de la langue ${ }^{28}$ :

«La langue n'est pas moins que la parole un objet de nature concrète, et c'est un grand avantage pour l'étude. Les signes linguistiques, pour être essentiellement psychiques, ne sont pas des abstractions: les associations ratifiées par le consentement collectif, et dont l'ensemble constitue la langue, sont des réalités qui ont leur siège dans le cerveau. »

Ces réalités, il appartient au linguiste de les expliciter, étant entendu que tout ce qu'il peut établir de rapports associatifs n'est que la redécouverte, quelque «prise de conscience » de ce que les sujets parlants savent inconsciemment ${ }^{29}$ :

« On peut dire que la somme des classements conscients et méthodiques faits par le grammairien qui étudie un état de langue sans faire intervenir l'histoire doit coïncider avec la somme des associations, conscientes ou non, mises en jeu dans la parole.»

Qu'on pense à la maïeutique socratique ou à l'épiphanie analytique, la chose est dite. Saussure est l'un des premiers pour qui la connaissance intuitive des sujets parlants soit une forme authentique du savoir. Les classements pratiques des sujets parlants ne sont plus un obstacle, un obscurcissement de l'énigme que la langue pose au linguiste aussi mais ils constituent le seul principe de réalité qui vaille en ces matières. Pour Saussure, le réel se confond avec la conscience (et l'inconscient) des sujets parlants -partant, il n'y a d'autre réalité, pour un linguiste, que ce savoir des locuteurs, même s'il ne se sait pas. Il ne lui reste qu'à écouter les locuteurs ${ }^{30}$ :

«Dès qu'on raisonne ces "abstractions", on voit la part de réalité qu'elles représentent, et un correctif très simple suffit pour donner à ces artifices du grammairien un sens légitime et exact. C'est ce qu'on a essayé de faire plus haut, en montrant que, unie par un lien intérieur à l'analyse subjective de la langue vivante, l'analyse objective a une place légitime et déterminée dans la méthode linguistique.»

Pourtant, ce « lien intérieur » ne se laisse pas si facilement serrer ${ }^{31}$ :

«Sans doute les sujets parlants ne connaissent pas ces difficultés; tout ce qui est significatif à un degré quelconque leur apparaît comme un élément concret, et ils le distinguent infailliblement dans le discours. Mais autre chose est de sentir ce jeu rapide et délicat des unités, autre chose d'en rendre compte par une analyse méthodique." 
"Toutes ces choses existent dans la langue, mais à titre d'entités abstraites; leur étude est difficile, parce qu'on ne peut savoir exactement si la conscience des sujets parlants va toujours aussi loin que les analyses du grammairien. » Saussure écarter l'expérimentation, l'introspection (fût-ce sur soi-même) en ce qu'elles n'auraient de pertinence que pour un point de vue particulier, non universalisable. Et que dire des enquêtes de terrain sinon rappeler la prodigieuse indifférence de Saussure pour les travaux de son compatriote Gilliéron. Pas d'autre quête du témoin que cette correspondance avec Giovanni Pascoli, à propos d'anagrammes...

\section{Ce que savent les sujets s'énonce dans la langue}

Puisque la parole, jamais, ne prouve rien en fait de langue, que toute enquête semble superflue, c'est que la langue a déjà, inscrit en elle, l'ensemble des rapports associatifs collectivement ratifiés et construits en dehors de toute volonté individuelle. Ce qui, pour le linguiste, objective le savoir des sujets, et qui permet de faire l'économie des corpus, ce sont les marques, qui se donnent à lire dans la langue, d'un savoir des sujets qui s'y est " précipité », pour en emprunter l'image à la chimie. D'où l'insistance de Saussure sur des processus qui réagencent les rapports associatifs, comme l'analogie, la composition (et la décomposition), l'étymologie populaire.

Cependant le travail du linguiste ne se réduit pas à l'enregistrement des effets de ces procédés ${ }^{32}$ :

«C'est un danger, en linguistique, de mêler les décompositions faites à différents points de vue avec celles faites par la langue; mais il est bon d'en faire le parallèle [et de confronter les procédés du grammairien pour décomposer le mot dans ses unités avec les procédés des sujets parlants.] Par cette opposition, on pourra mieux définir jusqu'où va l'analyse intérieure et instinctive. »

41 Si l'on s'en tient à la lettre de Saussure, la langue exhibe le travail réflexif des sujets parlants sur leur langue, travail effectué à leur insu puisque, au rebours de la parole, la langue est un « produit social » qui échappe à la volonté et à l'intelligence (Cours p. 30) :

«La parole est au contraire [de la langue] un acte individuel de volonté et

d'intelligence. »

Ce qu'on appelle l'arbitraire du signe, et qui a fait couler tant d'encre, porte, dans la violence que suggère ce mot, l'image d'une maîtrise absolue, l'imposition aux sujets de quelque chose qui les domine. Car, non seulement l'union du signifiant et du signifié ne se raisonne pas mais le système, fondé sur des différences relatives, oppositives et négatives, s'établit comme somme indéfinie et non hiérarchisée qu'un sujet parlant, parce qu'il s'inscrit du côté de la parole, se condamne à manquer comme totalité.

En récusant le concept associationniste d'association, Saussure dissout les fantasmes de maîtrise (un être de raison, libre de ses choix, face aux stimulations du monde et du moi); en déterminant qu'au principe des rapports associatifs, il y a un point de vue aussi particulier qu'on voudra, il refuse les normalisations et les rappels à l'ordre d'une psychologie de la conformité, voire du conformisme. Aux psychologues, il propose la tâche inouïe d'une sémiologie centrée sur le signifiant et privée de la fiction de facilité d'un homoncule ${ }^{33}$ : 
«(...) il n'y a jamais préméditation, ni même de méditation, de réflexion sur les formes, en dehors de l'acte, de l'occasion de la parole, sauf une activité inconsciente presque passive, en tout cas non créatrice : l'activité de classement. »

Peut-être les langues se suffiraient-elles de sujets, la conscience pouvant être donnée, comme la parole, par surcroît ${ }^{34}$ :

"Quelqu'un qui dort a en lui toute une langue (c'est la langue, cet ensemble qui a reçu la consécration sociale). »

On retrouverait la conclusion de Starobinski ${ }^{35}$ :

«La question était: qu'y a-t-il immédiatement derrière le vers ? la réponse n'est pas : le sujet créateur, mais : le mot inducteur. Non que Saussure aille jusqu'à effacer le rôle de la subjectivité de l'artiste : il lui semble toutefois qu'elle ne peut produire son texte qu'après passage par un pré-texte. Peut-être y a-t-il dans cette question un désir délibéré d'éluder tout problème relatif à une conscience créatrice. »

Au demeurant, Saussure nous avait prévenus ${ }^{36}$, alors qu'il constituait l'opposition langue/ parole :

«La langue n'est pas une fonction du sujet parlant, elle est le produit que l'individu enregistre passivement ; elle ne suppose jamais de préméditation, et la réflexion n'y intervient que pour l'activité de classement dont il sera question p. $170 \mathrm{sv}$. »

Que la lecture soit exégétique, historique ou polémique, la conclusion s'affiche contre tout structuralisme abstrait postulant l'auto-ordonnancement de la langue, et contre toute théorie narcissique de l'émerveillement devant une pensée humaine prélablement « libérée » de la dimension signifiante.

\section{BIBLIOGRAPHIE}

ARRIVE Michel (1986) : Linguistique et psychanalyse, Méridiens-Klincksieck, Paris

BENVENISTE Emile (1966) : Problèmes de linguistique générale, Gallimard, Paris

BERGOUNIOUX Gabriel (1992) : «La pathologie du langage entre les lettres et la médecine » in «Les débuts des sciences de l'homme », nº 54 de Communications, pp. 229-239, Seuil, Paris

BOUQUET Simon (1989) : «Le Cours de linguistique générale de Saussure et la philosophie », in CHISS et NORMAND (1989), pp. 103-119

CAUSSAT Pierre (1989) : « Une autorité verrouillante : Wilhelm Wundt (1832-1920)», in CHISS \& NORMAND (1989), pp. 67-90

CHARCOT Jean-Martin (1884) : Differenti forme d'afasia, Vallardi, Milan

CHISS Jean-Louis \& NORMAND Claudine (dir.) (1989) : «Extension et limites des théories du langage (1880-1980) », Histoire Epistémologie Langage, vol. XI, 2

CHISS Jean-Louis et PUECH Christian (1989) : «Enonciation, interaction, conversation : les théories du langage entre le psychique et le social », in CHISS \& NORMAND (1989), pp. †7-36

EGGER Victor (1904) : La parole intérieure, Alcan, Paris (1ère édition en 1881) 
ENGLER Rudolf (1962) : «Théorie et critique d'un principe saussurien : l'arbitraire du signe » in Cahiers Ferdinand de Saussure ${ }^{\circ}$ 19, Droz, Genève pp. 5-66

FABIANI Jean-Louis (1988) Les Philosophes de la République, Minuit, Paris

FAUCONNIER Gilles (1991) « Subdivision cognitive » in « Sémantique cognitive », n 53 de Communications, Seuil, Paris, pp. 229-248

GADET Françoise (1990) Saussure, une science de la langue, PUF, Paris

GODEL Robert $\left(1969^{2}\right)$ : Les sources manuscrites du Cours de Linguistique Générale, Droz, Genève HORMANN Hans (1972) : Introduction à la psycholinguistique, Larousse, Paris

SAUSSURE Ferdinand de/ ENGLER Rudolf (1968 sq.) : Cours de linguistique générale, édition critique établie par Rudolf ENGLER, Otto Harrassowitz, Wiesbaden

SAUSSURE Ferdinand de (1973) : Cours de linguistique générale, édition critique préparée par Tullio DE MAURO, Payot, Paris

SECHEHAYE Albert (1908) : Programme et méthodes de la linguistique théorique. Psychologie du langage, Champion, Paris

STAROBINSKI Jean (1971) : Les mots sous les mots, Gallimard, Paris

THUMB A. et MARBE K. (1901) : Experimentelle Untersuchungen über die psychologischen Grundlagen der sprachlichen Analogiebildung, Engelmann, Leipzig

VAN GINNEKEN Jacob (1907) Principes de linguistique psychologique, Marcel Rivière, Paris/ E. Van der Vecht, Amsterdam/ Otto Harrassowitz, Leipzig

\section{NOTES}

1. Désormais sténographié Cours.

2. Caussat (1989)

3. Cahier de Mme Sechehaye, in Saussure/Engler (1968) p. 20. Comme souvent, il est difficle de déterminer si « psychologique » est à prendre au sens de " psychique » ou de « psychologique ».

4. Godel (1969) p. 48

5. Arrivé (1986)

6. Fabiani (1988)

7. Saussure/Engler (1968) p. 50

8. Ibid. p. 233. C'est nous qui soulignons dans les deux versions présentées, celle de Madame Sechehaye et celle de Francis Joseph respectivement.

9. Bergounioux (1992)

10. Benveniste (1966). Il utilise à la place « relation », " articulation », « rapports ».

11. Saussure / Engler (1968) p. 25 ; notes de Riedlinger

12. Saussure/Engler (1968), p. 278. Notes du troisième cours recueillies par Emile Constantin.

13. La localisation de l'association dans le cerveau (la "psyché ») est une façon de marquer la différence du signe et du symbole. Dans le symbole, l'association, livrée d'emblée par un « rudiment de lien naturel », ne présuppose pas un circuit de pensée (Cours, p. 101).

14. Cours, p. 156 et Saussure/Engler (1968) p. 36: "union d'un genre particulier». On peut méditer l'opposition distinctive des radicaux dans les termes opposés, entre la racine -sociprésente dans « association » et l'un- de « union ».

15. Notamment, p. 29. 
16. Saussure/Engler (1968) p. 43, « Notes sur Programme et méthodes de la linguistique théorique d'Albert Sechehaye ».

17. Cours, p. 189

18. Cours, p. 189. Notons que l'union du signifiant et du signifié ne peut constituer une association puisque -c'est le fondement de l'arbitraire du signe- le signifiant et le signifié n'ont de commun qu'une appartenance au "psychique" qui ne peut donner lieu, en soi, à aucune forme de catégorisation, sinon quelque motivation relative.

19. Saussure/Engler (1968), pp. 280-282

20. Les sources manuscrites témoignent d'une hésitation entre cette désignation et " groupes", "groupes associatifs», « groupements», « séries associatives», «familles », "trésor », « magasin "...

21. Cours (p. 174). A la suite, Saussure hiérarchise les propriétés: «Cependant, de ces deux caractères de la série associative, ordre indéterminé et nombre indéfini, seul le premier se vérifie toujours; le second peut manquer.

22. Notamment les tests d' " associations de mots ", à distinguer de ce que Freud a promu comme " association libre » et qui était, du discours de l'inconscient, une autre façon de rompre avec la visée d'évaluation et d'uniformisation du behaviourisme.

23. Dont il est à craindre qu'une notion aussi centrale pour le cognitivisme que celle de prototype ne soit la version soft et ultra-moderne.

24. Cours, p. 174

25. Benveniste (1966) p. 39

26. Cours, p. 150-151

27. Saussure/Engler p. 280

28. Cours p. 32

29. Cours p. 189. "Inconsciemment » ne saurait équivoquer avec ce que Freud promeut sous ce vocable.

30. Cours p. 253

31. Cours p. 148 et p. 190

32. Saussure/Engler (1968) p. 419

33. Godel, p. 145

34. Saussure/Engler (1968) p. 98

35. Starobinski (1971) p. 152-153

36. Cours p. 30 